\title{
Lepton polarization and $\mathrm{CP}$-violating effects in $B \rightarrow K^{*} \tau^{+} \tau^{-}$decay in standard and two Higgs doublet models
}

\author{
T. M. Aliev *, M. Savcl ${ }^{\dagger}$ \\ Physics Department, Middle East Technical University \\ 06531 Ankara, Turkey
}

\begin{abstract}
The most general model independent expressions for the CP-violating asymmetry, longitudinal, transversal and normal polarizations of leptons are derived. Application of these general results to the concrete models such as Standard model and three different types of two Higgs doublet model is discussed.
\end{abstract}

*e-mail: taliev@metu.edu.tr

†e-mail: savci@metu.edu.tr 


\section{Introduction}

Rare $B$ meson decays, induced by flavor-changing neutral current (FCNC) $b \rightarrow s$ transitions, is one of the most promising research area in high energy physics. Theoretical interest to the rare $B$ decays lies in their role as a potential precision testing ground for the standard model (SM) at loop level and experimentally these decays will provide quantitative information about the Cabibbo-Kobayashi-Maskawa (CKM) matrix elements $V_{t d}$, $V_{t s}$ and $V_{t b}$. Besides, these rare decays have the potential of establishing new physics beyond SM, such as two Higgs doublet model (2HDM), minimal supersymmetric extension of the SM (MSSM), left-right models, etc.

At present, the main interest is focused on the rare $B$ meson decays, for which the SM predicts "large" branching ratios and that can be potentially measurable at working $B$ factories and LHC. The rare $B \rightarrow K^{*} \ell^{+} \ell^{-}(\ell=e, \mu, \tau)$ decays are such ones. At the same time this decay constitutes a suitable tool in looking for new physics beyond the SM. At quark level the process is described by the $b \rightarrow s \ell^{+} \ell^{-}$transition. This transition in framework of the SM and its various extensions have been extensively investigated [1]-[15].

One efficient way in establishing new physics is the measurement of lepton polarization. This problem is widely discussed in literature for the $b \rightarrow s \ell^{+} \ell^{-}$decay [16- 19]. Note that all previous studies for the lepton polarization, except the work [19, have been limited to SM and its minimal extensions. In 19 the analysis of the $\tau$ lepton polarization for the $b \rightarrow s \tau^{+} \tau^{-}$decay was performed in a model independent way. In this work twelve (ten local and two nonlocal) four-Fermi operator interactions were introduced in a model independent way instead of three independent structures which are present in SM.

It is well known that the theoretical analysis of the inclusive decays is rather easy but their experimental detection is quite difficult. However for exclusive decays the situation is contrary to this case, i.e., their experimental study is easy but theoretical investigation is difficult. This is due to the fact that the description of the exclusive decays requires form factors, i.e., the matrix elements of the effective Hamiltonian between initial $B$ and final meson states. This problem is related to to the nonperturbative sector of the QCD and it can be solved only by means of a nonperturbative approach.

These matrix elements have been investigated in framework of different approaches such as chiral theory [20], three point QCD sum rules method [21], relativistic quark model by the light-front formalism [22], effective heavy quark theory 23] and light cone QCD sum rules [24, 25].

The aim of the present paper is to perform a comprehensive study the lepton polarizations and CP-violating asymmetry in the exclusive $B \rightarrow K^{*} \ell^{+} \ell^{-}(\ell=\mu, \tau)$ decay in the $\mathrm{SM}$ and three versions of the 2HDM. Note that this decay in framework of 2HDM (model I and model II) were investigated in [15] (second reference), where $P_{L}$ and $P_{T}$ and $A_{F B}$ were studied. Here in this work we extend these considerations by studying in model III, including normal polarization of $\tau$ lepton and CP-violating asymmetry, using the current limits for the parameters of $2 \mathrm{HDM}$ coming from low energy experiments like $B^{0}-\bar{B}^{0}$ mixing, $\rho$-parameter analysis and $b \rightarrow s \gamma$ decay.

The paper is organized as follows. In section 2, starting from a general form of fourFermi interactions we derive model independent expressions for the longitudinal, transversal and normal polarizations. In section 3, we apply the above-mentioned general results of 
the lepton polarizations to SM and to three types of 2HDM (so called models I, II and III). A brief summary of our results is presented in this section.

\section{Lepton polarizations}

In this section we present the expressions for the longitudinal, transversal and normal polarizations of the $\tau$ lepton in a model independent way. For this aim we follow [19 where the matrix element for $b \rightarrow s \tau^{+} \tau^{-}$transition is given in terms of twelve most general independent four-Fermi interactions:

$$
\begin{aligned}
\mathcal{M} & =\frac{G \alpha}{\sqrt{2} \pi} V_{t b} V_{t s}^{*}\left\{C_{S L} \bar{s} i \sigma_{\mu \nu} \frac{q^{\nu}}{q^{2}}\left(m_{s} L\right) b \bar{\ell} \gamma_{\mu} \ell+C_{B R} \bar{s} i \sigma_{\mu \nu} \frac{q^{\nu}}{q^{2}}\left(m_{b} R\right) b \bar{\ell} \gamma_{\mu} \ell\right. \\
& +C_{L L} \bar{s}_{L} \gamma_{\mu} b_{L} \bar{\ell}_{L} \gamma^{\mu} \ell_{L}+C_{L R} \bar{s}_{L} \gamma_{\mu} b_{L} \bar{\ell}_{R} \gamma^{\mu} \ell_{R}+C_{R L} \bar{s}_{R} \gamma_{\mu} b_{R} \bar{\ell}_{L} \gamma^{\mu} \ell_{L} \\
& +C_{R R} \bar{s}_{R} \gamma_{\mu} b_{R} \bar{\ell}_{R} \gamma^{\mu} \ell_{R}+C_{L R L R} \bar{s}_{L} b_{R} \bar{\ell}_{L} \ell_{R}+C_{R L L R} \bar{s}_{R} b_{L} \bar{\ell}_{L} \ell_{R} \\
& +C_{L R R L} \bar{s}_{L} b_{R} \bar{\ell}_{R} \ell_{L}+C_{R L R L} \bar{s}_{R} b_{L} \bar{\ell}_{R} \ell_{L}+C_{T} \bar{s} \sigma_{\mu \nu} b \bar{\ell} \sigma^{\mu \nu} \ell \\
& \left.+i C_{T E} \epsilon^{\mu \nu \alpha \beta} \bar{s} \sigma_{\mu \nu} b \bar{\ell} \sigma_{\alpha \beta} \ell\right\},
\end{aligned}
$$

where $C_{X X}$ are the coefficient of the four-Fermi interactions. Among them, there are two non-local Fermi interactions denoted by $C_{S L}$ and $C_{B R}$, which correspond to $-2 C_{7}^{e f f}$ in the SM. Two $\left(C_{L L}, C_{L R}\right)$ of the four vector type interactions $\left(C_{L L}, C_{L R}, C_{R L}\right.$ and $\left.C_{R R}\right)$ are also present in the SM (in the forms of $C_{L L}=C_{9}^{e f f}-C_{10}$ and $C_{L R}=C_{9}^{e f f}+C_{10}$ ). $C_{L R L R}$, $C_{R L L R}, C_{L R R L}$ and $C_{R L R L}$ are the scalar type interactions and the last two terms with coefficients $C_{T}$ and $C_{T E}$ are the tensor type interactions.

For simplicity we will take the mass of the strange quark to be zero and neglect tensor type interactions, since it was indicated in [26] that the physical observables are not sensitive to the presence of the tensor type interactions. Having established the matrix element for the $b \rightarrow s \tau^{+} \tau^{-}$transition, our next problem is calculation of the matrix elements, $\left\langle K^{*}\left|\bar{s} \gamma_{\mu}\left(1 \pm \gamma_{5}\right) b\right| B\right\rangle,\left\langle K^{*}\left|\bar{s} i \sigma_{\mu \nu} q^{\nu}\left(1+\gamma_{5}\right) b\right| B\right\rangle$ and $\left\langle K^{*}\left|\bar{s}\left(1 \pm \gamma_{5}\right) b\right| B\right\rangle$, in order to be able to calculate the physically measurable quantities at hadronic level. These matrix elements can be written in terms of the form factors in the following way:

$$
\begin{aligned}
& \left\langle K^{*}\left(p_{K^{*}}, \varepsilon\right)\left|\bar{s} \gamma_{\mu}\left(1 \pm \gamma_{5}\right) b\right| B\left(p_{B}\right)\right\rangle= \\
& \quad-\epsilon_{\mu \nu \rho \sigma} \varepsilon^{* \nu} p_{K^{*}}^{\rho} q^{\sigma} \frac{2 V\left(q^{2}\right)}{m_{B}+m_{K^{*}}} \pm i \varepsilon_{\mu}^{*}\left(m_{B}+m_{K^{*}}\right) A_{1}\left(q^{2}\right) \mp i\left(p_{B}+p_{K^{*}}\right)_{\mu}\left(\varepsilon^{*} q\right) \frac{A_{2}\left(q^{2}\right)}{m_{B}+m_{K^{*}}} \\
& \quad \mp i q_{\mu} \frac{2 m_{K^{*}}}{q^{2}}\left(\varepsilon^{*} q\right)\left[A_{3}\left(q^{2}\right)-A_{0}\left(q^{2}\right)\right] \\
& \left\langle K^{*}\left(p_{K^{*}}, \varepsilon\right)\left|\bar{s} i \sigma_{\mu \nu} q^{\nu}\left(1+\gamma_{5}\right) b\right| B\left(p_{B}\right)\right\rangle= \\
& \quad 4 \epsilon_{\mu \nu \rho \sigma} \varepsilon^{* \nu} p_{K^{*}}^{\rho} q^{\sigma} T_{1}\left(q^{2}\right)+2 i\left[\varepsilon_{\mu}^{*}\left(m_{B}^{2}-m_{K^{*}}^{2}\right)-\left(p_{B}+p_{K^{*}}\right)_{\mu}\left(\varepsilon^{*} q\right)\right] T_{2}\left(q^{2}\right) \\
& \quad+2 i\left(\varepsilon^{*} q\right)\left[q_{\mu}-\left(p_{B}+p_{K^{*}}\right)_{\mu} \frac{q^{2}}{m_{B}^{2}-m_{K^{*}}^{2}}\right] T_{3}\left(q^{2}\right)
\end{aligned}
$$


where $\varepsilon$ is the polarization vector of $K^{*}$ meson and $q=p_{B}-p_{K^{*}}$ is the momentum transfer. In order to ensure finiteness of $(2)$ at $q^{2}=0$, we assume that $A_{3}\left(q^{2}=0\right)=A_{0}\left(q^{2}=0\right)$. To calculate the matrix element $\left\langle K^{*}\left|\bar{s}\left(1 \pm \gamma_{5}\right) b\right| B\right\rangle$, we multiply both sides of Eq. (2) by $q_{\mu}$ and use the equation of motion. Neglecting the mass of the strange quark, we get

$$
\begin{aligned}
& \left\langle K^{*}\left(p_{K^{*}}, \varepsilon\right)\left|\bar{s}\left(1 \pm \gamma_{5}\right) b\right| B\left(p_{B}\right)\right\rangle=\frac{1}{m_{b}}\left\{\mp i\left(\varepsilon^{*} q\right)\left(m_{B}+m_{K^{*}}\right) A_{1}\left(q^{2}\right)\right. \\
& \left.\quad \pm i\left(m_{B}-m_{K^{*}}\right)\left(\varepsilon^{*} q\right) A_{2}\left(q^{2}\right) \pm 2 i m_{K^{*}}\left(\varepsilon^{*} q\right)\left[A_{3}\left(q^{2}\right)-A_{0}\left(q^{2}\right)\right]\right\} .
\end{aligned}
$$

Using the equation of motion, the form factor $A_{3}$ can be expressed as a linear combination of the form factors $A_{1}$ and $A_{2}$ (see 21])

$$
A_{3}\left(q^{2}\right)=\frac{m_{B}+m_{K^{*}}}{2 m_{K^{*}}} A_{1}\left(q^{2}\right)-\frac{m_{B}-m_{K^{*}}}{2 m_{K^{*}}} A_{2}\left(q^{2}\right) .
$$

Using this relation we obtain

$$
\left\langle K^{*}\left(p_{K^{*}}, \varepsilon\right)\left|\bar{s}\left(1 \pm \gamma_{5}\right) b\right| B\left(p_{B}\right)\right\rangle=\frac{1}{m_{b}}\left\{\mp 2 i m_{K^{*}}\left(\varepsilon^{*} q\right) A_{0}\left(q^{2}\right)\right\} .
$$

From Eqs. (1), (2), (3) and (6) we get the following expression for the matrix element of the $B \rightarrow K^{*} \ell^{+} \ell^{-}$decay

$$
\begin{aligned}
\mathcal{M} & =\frac{G \alpha}{4 \sqrt{2} \pi} V_{t b} V_{t s}^{*} \\
& \times\left\{\bar{\ell} \gamma_{\mu}\left(1-\gamma_{5}\right) \ell\left[-\epsilon_{\mu \nu \rho \sigma} \varepsilon^{* \nu} p_{K^{*}}^{\rho} q^{\sigma}\left(2 A_{1}\right)-i \varepsilon_{\mu}^{*} B_{1}+i\left(\varepsilon^{*} q\right)\left(p_{B}+p_{K^{*}}\right)_{\mu} B_{2}+i q_{\mu}\left(\varepsilon^{*} q\right) B_{3}\right]\right. \\
& +\bar{\ell} \gamma_{\mu}\left(1+\gamma_{5}\right) \ell\left[-\epsilon_{\mu \nu \rho \sigma} \varepsilon^{* \nu} p_{K^{*}}^{\rho} q^{\sigma}\left(2 C_{1}\right)-i \varepsilon_{\mu}^{*} D_{1}+i\left(\varepsilon^{*} q\right)\left(p_{B}+p_{K^{*}}\right)_{\mu} D_{2}+i q_{\mu}\left(\varepsilon^{*} q\right) D_{3}\right] \\
& \left.+\bar{\ell}\left(1-\gamma_{5}\right) \ell i\left(\varepsilon^{*} q\right) B_{4}+\bar{\ell}\left(1+\gamma_{5}\right) \ell i\left(\varepsilon^{*} q\right) B_{5}\right\}
\end{aligned}
$$

where

$$
\begin{aligned}
A_{1} & =\left(C_{L L}+C_{R L}\right) \frac{V\left(q^{2}\right)}{m_{B}+m_{K^{*}}}-2 C_{B R} \frac{m_{b}}{q^{2}} T_{1}, \\
B_{1} & =\left(C_{L L}-C_{R L}\right)\left(m_{B}+m_{K^{*}}\right) A_{1}-2 C_{B R} \frac{m_{b}}{q^{2}}\left(m_{B}^{2}-m_{K^{*}}^{2}\right) T_{2}, \\
B_{2} & =\frac{C_{L L}-C_{R L}}{m_{B}+m_{K^{*}}} A_{2}-2 C_{B R} \frac{m_{b}}{q^{2}}\left[T_{2}+\frac{q^{2}}{m_{B}^{2}-m_{K^{*}}^{2}} T_{3}\right], \\
B_{3} & =\left(C_{L L}-C_{R L}\right) \frac{2 m_{K^{*}}}{q^{2}}\left(A_{3}-A_{0}\right)-2 C_{B R} \frac{m_{b}}{q^{2}} T_{3}, \\
C_{1} & =A_{1}\left(C_{L L} \rightarrow C_{L R}, \quad C_{R L} \rightarrow C_{R R}\right), \\
D_{1} & =B_{1}\left(C_{L L} \rightarrow C_{L R}, \quad C_{R L} \rightarrow C_{R R}\right), \\
D_{2} & =B_{2}\left(C_{L L} \rightarrow C_{L R}, \quad C_{R L} \rightarrow C_{R R}\right), \\
D_{3} & =B_{3}\left(C_{L L} \rightarrow C_{L R}, \quad C_{R L} \rightarrow C_{R R}\right),
\end{aligned}
$$




$$
\begin{aligned}
& B_{4}=-\left(C_{L R R L}-C_{R L R L}\right)\left(\frac{2 m_{K^{*}}}{m_{b}} A_{0}\right), \\
& B_{4}=-\left(C_{L R L R}-C_{R L L R}\right)\left(\frac{2 m_{K^{*}}}{m_{b}} A_{0}\right),
\end{aligned}
$$

At this point we would like to make the following comment. The main difference from the SM case is, we have six different structures (after setting $m_{s}=0$ and neglecting tensor interactions) in the inclusive channel, while under the same conditions we have only two new structures, namely scalar type interactions proportional to $B_{4}$ and $B_{5}$. On the other hand there appears no any new structure in the 2HDM.

Having established this matrix element, let us now consider the final lepton polarization. We define the following three orthogonal unit vectors:

$$
\begin{aligned}
\vec{e}_{L} & =\frac{\vec{p}_{1}}{\left|\vec{p}_{1}\right|}, \\
\vec{e}_{N} & =\frac{\vec{p}_{K^{*}} \times \vec{p}_{1}}{\left|\vec{p}_{K^{*}} \times \vec{p}_{1}\right|}, \\
\vec{e}_{T} & =\vec{e}_{L} \times \vec{e}_{N},
\end{aligned}
$$

where $\vec{p}_{1}$ and $\vec{p}_{K^{*}}$ are the three-momenta of the lepton $\ell^{-}$and $K^{*}$ meson, respectively, in the center of mass of final leptons. The differential decay rate for any spin direction $\vec{n}$ of the $\ell^{-}$lepton, where $\vec{n}$ is a unit vector in the $\ell^{-}$rest frame, can be expressed in the following form

$$
\frac{d \Gamma(\vec{n})}{d q^{2}}=\frac{1}{2}\left(\frac{d \Gamma}{d q^{2}}\right)_{0}\left[1+\left(P_{L} \vec{e}_{L}+P_{N} \vec{e}_{N}+P_{T} \vec{e}_{T}\right) \cdot \vec{n}\right],
$$

where the subscript " 0 " corresponds to the unpolarized differential decay rate whose explicit form will be presented below. $P_{L}, P_{N}$ and $P_{T}$ are recognized as the longitudinal, normal and transversal polarizations, respectively. It follows from the definition of unit vectors $\vec{e}_{i}$ that $P_{T}$ obviously lies in the decay plane whose orientation is determined by the vectors $\vec{p}_{1}$ and $\vec{p}_{K^{*}}$ and $P_{N}$ is perpendicular to this plane.

The expression for the unpolarized differential decay rate in Eq. (10) can be written as:

$$
\begin{aligned}
\left(\frac{d \Gamma}{d q^{2}}\right)_{0} & =\frac{G^{2} \alpha^{2}}{2^{14} \pi^{5} m_{B}}\left|V_{t b} V_{t s}^{*}\right|^{2} \lambda^{1 / 2} v \\
& \times\left\{32 \lambda m_{B}^{4}\left[\frac{1}{3}\left(m_{B}^{2} s-m_{\ell}^{2}\right)\left(\left|A_{1}\right|^{2}+\left|C_{1}\right|^{2}\right)+2 m_{\ell}^{2} \operatorname{Re}\left(A_{1} C_{1}^{*}\right)\right]\right. \\
& +96 m_{\ell}^{2} \operatorname{Re}\left(B_{1} D_{1}^{*}\right)-\frac{4}{r} m_{B}^{2} m_{\ell} \lambda \operatorname{Re}\left[\left(B_{1}-D_{1}\right)\left(B_{4}^{*}-B_{5}^{*}\right)\right] \\
& \left.+\frac{8}{r} m_{B}^{2} m_{\ell}^{2} \lambda\left\{\operatorname{Re}\left[\left(B_{3}^{*}+D_{2}^{*}-D_{3}^{*}\right) B_{1}\right]+\operatorname{Re}\left[\left(B_{2}^{*}-B_{3}^{*}+D_{3}^{*}\right) D_{1}\right]-\operatorname{Re}\left(B_{4} B_{5}^{*}\right)\right]\right\} \\
& +\frac{4}{r} m_{B}^{4} m_{\ell}(1-r) \lambda\left\{\operatorname{Re}\left[\left(B_{2}-D_{2}\right)\left(B_{4}^{*}-B_{5}^{*}\right)\right]\right\} \\
& +\frac{8}{r} m_{B}^{4} m_{\ell}^{2}(1-r) \lambda\left\{\operatorname{Re}\left[-\left(B_{2}-D_{2}\right)\left(B_{3}^{*}-D_{3}^{*}\right)\right]\right\}
\end{aligned}
$$




$$
\begin{aligned}
& -\frac{8}{r} m_{B}^{4} m_{\ell}^{2} \lambda(2+2 r-s) \operatorname{Re}\left(B_{2} D_{2}^{*}\right)-\frac{4}{r} m_{B}^{4} m_{\ell} s \lambda \operatorname{Re}\left[\left(B_{3}-D_{3}\right)\left(B_{4}^{*}-B_{5}^{*}\right)\right] \\
& -\frac{4}{r} m_{B}^{4} m_{\ell}^{2} s \lambda\left[\left|B_{3}\right|^{2}+\left|D_{3}\right|^{2}-2 \operatorname{Re}\left(B_{3} D_{3}^{*}\right)\right]+\frac{2}{r} m_{B}^{2}\left(m_{B}^{2}-2 m_{\ell}^{2}\right) \lambda\left[\left|B_{4}\right|^{2}+\left|B_{5}\right|^{2}\right] \\
& -\frac{8}{3 r s} m_{B}^{2} \lambda\left[m_{\ell}^{2}(2-2 r+s)+m_{B}^{2} s(1-r-s)\right]\left[\operatorname{Re}\left(B_{1} B_{2}^{*}\right)+\operatorname{Re}\left(D_{1} D_{2}^{*}\right)\right] \\
& +\frac{4}{r s}\left[2 m_{\ell}^{2}(\lambda-6 r s)+m_{B}^{2} s(\lambda+12 r s)\right]\left[\left|B_{1}\right|^{2}+\left|D_{1}\right|^{2}\right] \\
& \left.+\frac{4}{3 r s} m_{B}^{4} \lambda\left\{m_{B}^{2} s \lambda+m_{\ell}^{2}[2 \lambda+3 s(2+2 r-s)]\right\}\left[\left|B_{2}\right|^{2}+\left|D_{2}\right|^{2}\right]\right\}
\end{aligned}
$$

The polarizations $P_{L}, P_{N}$ and $P_{T}$ are defined as:

$$
P_{i}\left(q^{2}\right)=\frac{\frac{d \Gamma}{d q^{2}}\left(\vec{n}=\vec{e}_{i}\right)-\frac{d \Gamma}{d q^{2}}\left(\vec{n}=-\vec{e}_{i}\right)}{\frac{d \Gamma}{d q^{2}}\left(\vec{n}=\vec{e}_{i}\right)+\frac{d \Gamma}{d q^{2}}\left(\vec{n}=-\vec{e}_{i}\right)} .
$$

After lengthy calculations we get the following general expressions for the longitudinal, transversal and normal polarizations of the $\ell^{-}$lepton (for $m_{s}=0$ and neglecting the tensor interaction)

$$
\begin{aligned}
P_{L} & =\frac{1}{\Delta} v\left\{\frac{4}{3 r} \lambda^{2} m_{B}^{6}\left[\left|B_{2}\right|^{2}-\left|D_{2}\right|^{2}\right]+\frac{4}{r} \lambda m_{B}^{2} m_{\ell} \operatorname{Re}\left[\left(B_{1}-D_{1}\right)\left(B_{4}^{*}+B_{5}^{*}\right)\right]\right. \\
& -\frac{4}{r} \lambda m_{B}^{4} m_{\ell}(1-r) \operatorname{Re}\left[\left(B_{2}-D_{2}\right)\left(B_{4}^{*}+B_{5}^{*}\right)\right]+\frac{32}{3} \lambda m_{B}^{6} s\left[\left|A_{1}\right|^{2}-\left|C_{1}\right|^{2}\right] \\
& -\frac{2}{r} \lambda m_{B}^{4} s\left[\left|B_{4}\right|^{2}-\left|B_{5}\right|^{2}\right]+\frac{4}{r} \lambda m_{B}^{4} m_{\ell} s \operatorname{Re}\left[\left(B_{3}-D_{3}\right)\left(B_{4}^{*}+B_{5}^{*}\right)\right] \\
& \left.-\frac{8}{3 r} \lambda m_{B}^{4}(1-r-s)\left[\operatorname{Re}\left(B_{1} B_{2}^{*}\right)-\operatorname{Re}\left(D_{1} D_{2}^{*}\right)\right]+\frac{4}{3 r} \lambda m_{B}^{2}(\lambda+12 r s)\left[\left|B_{1}\right|^{2}-\left|D_{1}\right|^{2}\right]\right\}, \\
P_{T} & =\frac{1}{\Delta} \sqrt{\lambda} \pi\left\{-8 m_{B}^{3} m_{\ell} \sqrt{s} \operatorname{Re}\left[\left(A_{1}+C_{1}\right)\left(B_{1}^{*}+D_{1}^{*}\right)\right]\right. \\
& +\frac{1}{2 r} m_{B}^{3} m_{\ell}(1+3 r+s) \sqrt{s}\left[2 \operatorname{Re}\left(B_{1} D_{2}^{*}\right)-2 \operatorname{Re}\left(B_{2} D_{1}^{*}\right)\right] \\
& +\frac{1}{r \sqrt{s}} m_{B} m_{\ell}(1-r-s)\left[\left|B_{1}\right|^{2}-\left|D_{1}\right|^{2}\right] \\
& +\frac{1}{r \sqrt{s}} m_{B} m_{\ell}^{2}(1-r-s)\left[2 \operatorname{Re}\left(B_{1} B_{5}^{*}\right)-2 \operatorname{Re}\left(D_{1} B_{4}^{*}\right)\right] \\
& +\frac{1}{2 r} m_{B}^{3} m_{\ell}(1-r-s) \sqrt{s} \operatorname{Re}\left[2\left(B_{1}+D_{1}\right)\left(B_{3}^{*}-D_{3}^{*}\right)\right] \\
& +\frac{1}{r \sqrt{s}} m_{B}^{3} m_{\ell}^{2} \lambda\left[-2 \operatorname{Re}\left(B_{2} B_{5}^{*}\right)+2 \operatorname{Re}\left(D_{2} B_{4}^{*}\right)\right] \\
& +\frac{1}{r \sqrt{s}} m_{B}^{5} m_{\ell}(1-r) \lambda\left[\left|B_{2}\right|^{2}-\left|D_{2}\right|^{2}\right]+\frac{1}{2 r} m_{B}^{5} m_{\ell} \lambda \sqrt{s} \operatorname{Re}\left[-2\left(B_{2}+D_{2}\right)\left(B_{3}^{*}-D_{3}^{*}\right)\right] \\
& +\frac{1}{2 r \sqrt{s}} m_{B}^{3} m_{\ell}[(1-r-s)(1-r)+\lambda]\left[-2 \operatorname{Re}\left(B_{1} B_{2}^{*}\right)+2 \operatorname{Re}\left(D_{1} D_{2}^{*}\right)\right]
\end{aligned}
$$




$$
\begin{aligned}
& +\frac{1}{2 r \sqrt{s}} m_{B}(1-r-s)\left(-2 m_{\ell}^{2}+m_{B}^{2} s\right)\left[2 \operatorname{Re}\left(D_{1} B_{5}^{*}\right)-2 \operatorname{Re}\left(B_{1} B_{4}^{*}\right)\right] \\
& \left.+\frac{1}{2 r \sqrt{s}} m_{B}^{3} \lambda\left(-2 m_{\ell}^{2}+m_{B}^{2} s\right)\left[-2 \operatorname{Re}\left(D_{2} B_{5}^{*}\right)+2 \operatorname{Re}\left(B_{2} B_{4}^{*}\right)\right]\right\} \\
P_{N} & =\frac{1}{\Delta} \pi v m_{B}^{3} \sqrt{\lambda} \sqrt{s}\left\{8 m_{\ell} \operatorname{Im}\left(B_{1}^{*} C_{1}+A_{1}^{*} D_{1}\right)\right. \\
& +\frac{1}{r} m_{B}^{2} \lambda\left[\operatorname{Im}\left(m_{\ell} B_{3}-m_{\ell} D_{3}-B_{4}\right) B_{2}^{*}+\operatorname{Im}\left(-B_{5}-B_{3}+D_{3}\right) D_{2}^{*}\right] \\
& +\frac{1}{r} m_{\ell}(1+3 r-s) \operatorname{Im}\left[\left(D_{1}+B_{1}\right)\left(B_{2}^{*}-D_{2}^{*}\right)\right] \\
& \left.+\frac{1}{r}(1-r-s)\left[\operatorname{Im}\left(B_{4}-m_{\ell} B_{3}+m_{\ell} D_{3}\right) B_{1}^{*}+\operatorname{Im}\left(m_{\ell} B_{3}-B_{5}-m_{\ell} D_{3}\right) D_{1}^{*}\right]\right\},
\end{aligned}
$$

where $\Delta$ is the expression within the curly parenthesis of the unpolarized differential decay rate in Eq. (11). These expressions for the longitudinal, transversal and normal polarizations are general and model independent (if the tensor interaction is neglected). It follows from the expressions of $P_{T}$ and $P_{N}$ that they are proportional to the lepton mass and therefore they are nonvanishing only for the $\tau$ lepton. In this work we also analyze the $\mathrm{CP}$-violating asymmetry, which is defined as

$$
A_{C P}\left(q^{2}\right)=\frac{\left(\frac{d \Gamma}{d q^{2}}\right)_{0}-\left(\frac{d \bar{\Gamma}}{d q^{2}}\right)_{0}}{\left(\frac{d \Gamma}{d q^{2}}\right)_{0}+\left(\frac{d \bar{\Gamma}}{d q^{2}}\right)_{0}},
$$

where $\left(d \Gamma / d q^{2}\right)_{0}$ is the unpolarized differential decay rate given by Eq. (11) and $\left(d \bar{\Gamma} / d q^{2}\right)_{0}$ is the unpolarized differential decay rate for the antiparticle channel. Note that in SM , CPviolating asymmetry is equal to zero (or suppressed very strongly), since all form factors are real (see below), Wilson coefficients $C_{7}^{\text {eff }}$ and $C_{10}$ are real and only $C_{9}^{\text {eff }}$ contains a strong phase. But this strong phase can not lead to $\mathrm{CP}$-violation itself. Using these general expressions we can study the sensitivity of the $\tau$-lepton polarizations on the new Wilson coefficients. Furthermore one can investigate how strongly these polarizations deviate from the SM predictions and for which Wilson coefficient this departure is more essential. But in the present work we will apply these general results to concrete models, namely to the SM and three type of $2 \mathrm{HDM}$, i.e., models I, II (about models I and II, see for example [27] and III. Note that in models I and II, the flavor changing neutral currents which appear at tree level are avoided by imposing ad hoc symmetry [28]. The phenomenological consequence of the 2HDM without this discrete symmetry has been investigated in [29] (see also [30]-39]). One novel feature of model III is existence of new weak phase which appears in Yukawa interaction of fermions with Higgs fields (see below). Existence of this new weak phase can lead to sizeable CP violation in $B \rightarrow K^{*} \ell^{+} \ell^{-}$decay. Therefore if in future experiments sizeable CP violation in the $B \rightarrow K^{*} \ell^{+} \ell^{-}$decay is discovered, it is an unambiguous indication of the existence of new physics beyond SM, since in the SM the $\mathrm{CP}$ asymmetry suppressed very strongly. 
Making the following replacements in the expressions given in Eq. (8), the explicit forms of $A_{i}, B_{i}, C_{i}$ and $D_{i}$ can be obtained in SM and 2HDM easily.

1. SM

$$
\begin{aligned}
C_{L L} & =C_{9}^{e f f}\left(m_{b}\right)-C_{10}\left(m_{b}\right), \\
C_{R L} & =0, \\
C_{B R} & =-2 C_{7}^{e f f}\left(m_{b}\right), \\
C_{L R} & =C_{9}^{e f f}\left(m_{b}\right)+C_{10}\left(m_{b}\right), \\
C_{R R} & =0, \\
C_{L R R L} & =C_{R L L R}=C_{L R L R}=C_{R L R L}=0 .
\end{aligned}
$$

2. $2 \mathrm{HDM}$

$$
\begin{aligned}
C_{L L} & =C_{9}^{e f f 2 H D M}\left(m_{b}\right)-C_{10}^{2 H D M}\left(m_{b}\right), \\
C_{R L} & =0 \\
C_{B R} & =-2 C_{7}^{e f f 2 H D M}\left(m_{b}\right), \\
C_{L R} & =C_{9}^{e f f 2 H D M}\left(m_{b}\right)+C_{10}^{2 H D M}\left(m_{b}\right), \\
C_{R R} & =0 \\
C_{L R R L} & =C_{Q_{1}}, \\
C_{R L R L} & =C_{Q_{2}}, \\
C_{L R L R} & =C_{Q_{1}}, \\
C_{R L L R} & =-C_{Q_{2}} .
\end{aligned}
$$

The coefficients $C_{i}^{2 H D M}\left(m_{W}\right)(i=7,9$ and 10) to the leading order are given by (see for example [40, 41])

$$
\begin{aligned}
C_{7}^{2 H D M}\left(m_{W}\right) & =x \frac{\left(7-5 x-8 x^{2}\right)}{24(x-1)^{3}}+\frac{x^{2}(3 x-2)}{4(x-1)^{4}} \ln x \\
& +\left|\lambda_{t t}\right|^{2}\left(\frac{y\left(7-5 y-8 y^{2}\right)}{72(y-1)^{3}}+\frac{y^{2}(3 y-2)}{12(y-1)^{4}} \ln y\right) \\
& +\lambda_{t t} \lambda_{b b}\left(\frac{y(3-5 y)}{12(y-1)^{2}}+\frac{y(3 y-2)}{6(y-1)^{3}} \ln y\right), \\
C_{9}^{2 H D M}\left(m_{W}\right) & =-\frac{1}{\sin ^{2} \theta_{W}} B\left(m_{W}\right)+\frac{1-4 \sin ^{2} \theta_{W}}{\sin ^{2} \theta_{W}} C\left(m_{W}\right) \\
& +\frac{x^{2}(25-19 x)}{36(x-1)^{3}}+\frac{-3 x^{4}+30 x^{3}-54 x^{2}+32 x-8}{18(x-1)^{4}} \ln x+\frac{4}{9} \\
& +\left|\lambda_{t t}\right|^{2}\left[\frac{1-4 \sin ^{2} \theta_{W}}{\sin ^{2} \theta_{W}} \frac{x y}{8}\left(\frac{1}{y-1}-\frac{1}{(y-1)^{2}} \ln y\right)\right.
\end{aligned}
$$




$$
\begin{aligned}
& \left.-y\left(\frac{47 y^{2}-79 y+38}{108(y-1)^{3}}-\frac{3 y^{3}-6 y^{3}+4}{18(y-1)^{4}} \ln y\right)\right], \\
C_{10}^{2 H D M}\left(m_{W}\right)= & \frac{1}{\sin ^{2} \theta_{W}}\left(B\left(m_{W}\right)-C\left(m_{W}\right)\right) \\
+ & \left|\lambda_{t t}\right|^{2} \frac{1}{\sin ^{2} \theta_{W}} \frac{x y}{8}\left(-\frac{1}{y-1}+\frac{1}{(y-1)^{2}} \ln y\right), \\
C_{Q_{1}}\left(m_{W}\right)= & \frac{m_{b} m_{\ell}}{m_{h^{0}}^{2}} \frac{1}{\left|\lambda_{t t}\right|^{2}} \frac{1}{\sin ^{2} \theta_{W}} \frac{x}{4}\left\{\left(\sin ^{2} \alpha+h \cos ^{2} \alpha\right) f_{1}(x, y)+\right. \\
+ & {\left[\frac{m_{h^{0}}^{2}}{m_{W}^{2}}+\left(\sin ^{2} \alpha+h \cos ^{2} \alpha\right)(1-z)\right] f_{2}(x, y)+} \\
+ & \left.\frac{\sin ^{2} 2 \alpha}{2 m_{H^{ \pm}}^{2}}\left[m_{h^{0}}^{2}-\frac{\left(m_{h^{0}}^{2}+m_{H^{0}}^{2}\right)^{2}}{2 m_{H^{0}}^{2}}\right] f_{3}(y)\right\}, \\
C_{Q_{2}}\left(m_{W}\right)= & \frac{m_{b} m_{\ell}}{m_{H^{ \pm}}^{2}} \frac{1}{\left|\lambda_{t t}\right|^{2}}\left\{f_{1}(x, y)+\left[1+\frac{m_{H^{ \pm}}^{2}-m_{A^{0}}^{2}}{m_{W}^{2}}\right] f_{2}(x, y)\right\},
\end{aligned}
$$

where

$$
\begin{aligned}
x & =\frac{m_{t}^{2}}{m_{W}^{2}}, \quad y=\frac{m_{t}^{2}}{m_{H^{ \pm}}^{2}}, \quad z=\frac{x}{y}, \quad h=\frac{m_{h^{0}}^{2}}{m_{H^{0}}^{2}}, \\
B(x) & =-\frac{x}{4(x-1)}+\frac{x}{4(x-1)^{2}} \ln x, \\
C(x) & =\frac{x}{4}\left(\frac{x-6}{2(x-1)}+\frac{3 x+2}{2(x-1)^{2}} \ln x\right), \\
f_{1}(x, y) & =\frac{x \ln x}{x-1}-\frac{y \ln y}{y-1}, \\
f_{2}(x, y) & =\frac{x \ln y}{(z-x)(x-1)}+\frac{\ln z}{(z-1)(x-1)}, \\
f_{3}(y) & =\frac{1-y+y \ln y}{(y-1)^{2}},
\end{aligned}
$$

$\sin ^{2} \theta_{W}=0.23$ is the Weinberg angle, $h^{0}, H^{0}$ and $A^{0}$ are two scalar and pseudoscalar Higgs fields, respectively. The coefficients $\lambda_{t t}$ and $\lambda_{b b}$ for model I and model II of the 2HDM are:

$$
\begin{array}{lll}
\lambda_{t t}=\cot \beta, & \lambda_{b b}=-\cot \beta, & \text { for model I }, \\
\lambda_{t t}=\cot \beta, & \lambda_{b b}=+\tan \beta, & \text { for model II },
\end{array}
$$

while in model III $\lambda_{t t}$ or $\lambda_{b b}$ is complex, i.e.,

$$
\lambda_{t t} \lambda_{b b} \equiv\left|\lambda_{t t} \lambda_{b b}\right| e^{i \phi}
$$


From Eqs. (16)-(20) we observe that the SM results for the Wilson coefficients $C_{7}^{S M}\left(m_{W}\right)$, $C_{9}^{S M}\left(m_{W}\right)$ amd $C_{10}^{S M}\left(m_{W}\right)$ (and correspondingly at $\mu=m_{b}$ scale) can all be obtained from 2HDM results by making the following replacements

$$
\begin{aligned}
& C_{Q_{1}} \rightarrow 0, \quad C_{Q_{2}} \rightarrow 0, \\
& C_{7}^{S M}\left(m_{W}\right)=C_{7}^{2 H D M}(y \rightarrow 0), \\
& C_{9}^{S M}\left(m_{W}\right)=C_{9}^{2 H D M}(y \rightarrow 0), \\
& C_{10}^{S M}\left(m_{W}\right)=C_{10}^{2 H D M}(y \rightarrow 0),
\end{aligned}
$$

The evolution of the Wilson coefficients from the higher scale $\mu=m_{W}$ down to the low energy scale $\mu=m_{b}$ is described by the renormalization group equation. The coefficients $C_{7}^{e f f}(\mu), C_{9}^{e f f}(\mu), C_{10}(\mu)$ at the scale $\mathcal{O}\left(\mu=m_{b}\right)$ are calculated in 42, 43, and $C_{Q_{1}}$ and $C_{Q_{2}}$ at the same scale to leading order are calculated in [41]. The Wilson coefficient $C_{10}$ is not modified as we move from $\mu=m_{W}$ to $\mu=m_{b}$ scale, i.e., $C_{10}\left(m_{b}\right) \equiv C_{10}^{2 H D M}\left(m_{W}\right)$. In order to calculate $C_{9}^{2 H D M}$ at $m_{b}$ scale, it is enough to make the replacement $C_{9}^{S M}\left(m_{W}\right) \rightarrow$ $C_{9}^{2 H D M}\left(m_{W}\right)$ and then solve the corresponding renormalization group equation. Hence, including the NLO QCD corrections, $C_{9}^{\text {eff }}\left(m_{b}\right)$ can be written as:

$$
\begin{aligned}
& C_{9}^{e f f}(\mu)=C_{9}^{2 H D M}(\mu)\left[1+\frac{\alpha_{s}(\mu)}{\pi} \omega(\hat{s})\right] \\
& \quad+g\left(\hat{m}_{c}, \hat{s}\right)\left[3 C_{1}(\mu)+C_{2}(\mu)+3 C_{3}(\mu)+C_{4}(\mu)+3 C_{5}(\mu)+C_{6}(\mu)\right] \\
& \quad-\frac{1}{2} g(0, \hat{s})\left(C_{3}(\mu)+3 C_{4}(\mu)\right)-\frac{1}{2} g(1, \hat{s})\left(4 C_{3}+4 C_{4}+3 C_{5}+C_{6}\right) \\
& \quad-\frac{1}{2} g(0, \hat{s})\left(C_{3}+3 C_{4}\right)+\frac{2}{9}\left(3 C_{3}+C_{4}+3 C_{5}+C_{6}\right),
\end{aligned}
$$

where $\hat{m}_{c}=m_{c} / m_{b}, \hat{s}=p^{2} / m_{b}^{2}$, and

$$
\begin{aligned}
\omega(\hat{s}) & =-\frac{2}{9} \pi^{2}-\frac{4}{3} L i_{2}(\hat{s})-\frac{2}{3} \ln (\hat{s}) \ln (1-\hat{s}) \\
& -\frac{5+4 \hat{s}}{3(1+2 \hat{s})} \ln (1-\hat{s})-\frac{2 \hat{s}(1+\hat{s})(1-2 \hat{s})}{3(1-\hat{s})^{2}(1+2 \hat{s})} \ln (\hat{s})+\frac{5+9 \hat{s}-6 \hat{s}^{2}}{3(1-\hat{s})(1+2 \hat{s})}
\end{aligned}
$$

represents the $\mathcal{O}\left(\alpha_{s}\right)$ correction from the one gluon exchange in the matrix element of $O_{9}$, while the function $g\left(\hat{m}_{c}, \hat{s}\right)$ arises from one loop contributions of the four-quark operators $\mathrm{O}_{1}-\mathrm{O}_{6}$, whose form is

$$
\begin{aligned}
g\left(y_{i}, \hat{s}\right)= & -\frac{8}{9} \ln \left(\hat{m}_{i}\right)+\frac{8}{27}+\frac{4}{9} y_{i} \\
& -\frac{2}{9}\left(2+y_{i}\right) \sqrt{\left|1-y_{i}\right|}\left\{\Theta\left(1-y_{i}\right)\left(\ln \frac{1+\sqrt{\left|1-y_{i}\right|}}{1-\sqrt{\left|1-y_{i}\right|}}-i \pi\right)\right. \\
& \left.+\Theta\left(y_{i}-1\right) 2 \arctan \frac{1}{\sqrt{y_{i}-1}}\right\},
\end{aligned}
$$

where $y_{i}=4 \hat{m}_{i}^{2} / \hat{p}^{2}$. The Wilson coefficient $C_{9}^{e f f}$ receives also long distance contributions, which have their origin in the real $c \bar{c}$ intermediate states, i.e., $J / \psi, \psi^{\prime}, \cdots$. The $J / \psi$ family 
is introduced by the Breit-Wigner distribution for the resonances through the replacement [3, 6]

$$
g\left(\hat{m}_{c}, \hat{s}\right) \rightarrow g\left(\hat{m}_{c}, \hat{s}\right)-\frac{3 \pi}{\alpha_{e m}^{2}} \kappa \sum_{V_{i}=J / \psi_{i}, \psi^{\prime}, \ldots} \frac{m_{V_{i}} \Gamma\left(V_{i} \rightarrow \ell^{+} \ell^{-}\right)}{\left(p^{2}-m_{V_{i}}^{2}\right)+i m_{V_{i}} \Gamma_{V_{i}}}
$$

where the phenomenological parameter $\kappa=2.3$ is chosen in order to reproduce correctly the experimental value of the branching ratio (see for example [44])

\section{Numerical analysis}

In this section we would like to present our numerical results. The main free parameters $\lambda_{t t}, \lambda_{b b}$ of the $2 \mathrm{HDM}$ are restricted from $B \rightarrow X_{s} \gamma$ decay, $B^{0}-\bar{B}^{0}$ mixing, $\rho$ parameter and neutron electric-dipole moment [36], that yields $\left|\lambda_{b b}\right|=50,\left|\lambda_{t t}\right| \leq 0.03$. Throughout the numerical analysis for the mass of the Higgs bosons we have used $m_{h^{0}}=80 \mathrm{GeV}, m_{H^{ \pm}}=$ $250 \mathrm{GeV}, m_{A^{0}}=250 \mathrm{GeV}$ and $m_{H^{0}}=150 \mathrm{GeV}$.

For the values of the form factors, we have used the results of [25], where the radiative corrections to the leading twist contribution and $S U(3)$ breaking effects are also taken into account. The $q^{2}$ dependence of the form factors can be represented in terms of three parameters as

$$
F\left(q^{2}\right)=\frac{F(0)}{1-a_{F} \frac{q^{2}}{m_{B}^{2}}+b_{F}\left(\frac{q^{2}}{m_{B}^{2}}\right)^{2}}
$$

where, the values of parameters $F(0), a_{F}$ and $b_{F}$ for the $B \rightarrow K^{*} \ell^{+} \ell^{-}$decay are listed in Table 1.

\begin{tabular}{|l|ccr|}
\hline & $F(0)$ & $a_{F}$ & $b_{F}$ \\
\hline$A_{1}^{B \rightarrow K^{*}}$ & $0.34 \pm 0.05$ & 0.60 & -0.023 \\
$A_{2}^{B \rightarrow K^{*}}$ & $0.28 \pm 0.04$ & 1.18 & 0.281 \\
$V^{B \rightarrow K^{*}}$ & $0.46 \pm 0.07$ & 1.55 & 0.575 \\
$T_{1}^{B \rightarrow K^{*}}$ & $0.19 \pm 0.03$ & 1.59 & 0.615 \\
$T_{2}^{B \rightarrow K^{*}}$ & $0.19 \pm 0.03$ & 0.49 & -0.241 \\
$T_{3}^{B \rightarrow K^{*}}$ & $0.13 \pm 0.02$ & 1.20 & 0.098 \\
\hline
\end{tabular}

Table 1: $B$ meson decay form factors in a three-parameter fit, where the radiative corrections to the leading twist contribution and $\mathrm{SU}(3)$ breaking effects are taken into account [25].

In Fig. (1) we present the dependence of the $\mathrm{CP}$-violating asymmetry on $q^{2}$ and on the weak phase $\phi$ for the $B \rightarrow K^{*} \tau^{+} \tau^{-}$decay in model III, since we have already noted that 
in SM and in models I and II the $\mathrm{CP}$-violating asymmetry is practically zero. We observe that $\mathrm{CP}$ asymmetry differs from zero in the region $0<\phi<2 \pi$, except at $\phi=0, \pi$ and $2 \pi$, and its value in the region $0<\phi<\pi(\pi<\phi<2 \pi)$ is negative (positive).

Fig .(2) depicts the dependence of the averaged CP asymmetry $\left\langle A_{C P}\right\rangle$ (here and in all of the following discussions, by the averaged values of the physical quantities we mean integration over $q^{2}$ in the region $\left.14 \mathrm{GeV}^{2} \leq q^{2} \leq\left(m_{B}-m_{K^{*}}\right)^{2}\right)$ on the weak phase angle $\phi$ in model III, taking into account short and long distance contributions. It follows from this figure that $\left\langle A_{C P}\right\rangle$ varies in the range $(-0.04,0.04)$ which is different from zero and it definitely is an indication of the existence of new physics beyond $\mathrm{SM}$, since $\left\langle A_{C P}\right\rangle$ is practically equal to zero in the SM.

In Fig. (3), the dependence of $P_{L}$ on $q^{2}$ and the weak phase angle $\phi$ without long distance effects in model III is presented. From this figure one can see that, for $q^{2}>14 \mathrm{GeV}^{2}, P_{L}$ varies in the range $(-0.65,-0.8)$ which is larger than the SM prediction. This is due to the fact that the "new" contribution which comes from the charged Higgs boson gives constructive interference to the SM results.

In Fig. (4) we present the averaged longitudinal polarization $\left\langle P_{L}\right\rangle$ on the weak phase angle $\phi$, taking into account short and long distance contributions. For completeness the predictions of SM, model I and model II on $\left\langle P_{L}\right\rangle$ are also presented. It is observed from this figure that $\left\langle P_{L}\right\rangle$ in model III as modulo, is larger than the ones predicted by SM, model I and model II. Therefore an observation of $\left|\left\langle P_{L}\right\rangle\right| \geq 0.65$ is another conclusive confirmation of the existence of new physics beyond SM.

In Figs. (5) and (6) we present the dependence of the transversal and normal polarizations of the $\tau$ lepton on $q^{2}$ and on the weak phase angle $\phi$, respectively, without long distance effects in model III. The dependence of the averaged transversal and normal polarizations on the weak phase angle, taking into account short and long distance contributions, are depicted in Figs. (7) and (8), respectively. For sake of completeness we presented also the predictions of SM, model I and model II of the same physical quantity in both figures. Fig. (7) clearly depicts that, the prediction of model III on $\left\langle P_{T}\right\rangle$ as modulo, is approximately five times smaller than the ones predicted by SM, model I and model II. However the situation is totally different for $\left\langle P_{N}\right\rangle$, having a range of values in the region $(0.10,0.15)$ in model III, it is approximately two or three times larger than the ones predicted by SM, model I and model II.

Here we would like to make the following remark. It follows from Eq. (13) that $P_{N}$ is defined as the imaginary part of the form factors and of the corresponding Wilson coefficients $C_{7}^{\text {eff }}, C_{9}^{\text {eff }}, C_{10}, C_{Q_{1}}$ and $C_{Q_{2}}$. In SM $C_{7}^{\text {eff }}$ and $C_{10}$ are real and only $C_{9}^{\text {eff }}$ has imaginary part. On the other side all theoretical methods predict these form factors to be real quantities. For this reason, if in future experiments a different value for $P_{N}$ were observed compared to the SM prediction, it is an indication of unambiguous information about the existence of the above-mentioned $\mathrm{CP}-$ violating phase in theory.

In conclusion, we have investigated the exclusive $B \rightarrow K^{*} \tau^{+} \tau^{-}$decay in the SM and in three different versions of the 2HDM. From the results we have obtained we conclude that the combined analysis of the $\mathrm{CP}$-violating asymmetry and $\tau$ lepton polarization effects are very useful tools in looking for new physics beyond SM. 


\section{References}

[1] W. -S. Hou, R. S. Willey and A. Soni, Phys. Rev. Lett. 58 (1987) 1608.

[2] N. G. Deshpande and J. Trampetic, Phys. Rev. Lett. 60 (1988) 2583.

[3] C. S. Lim, T. Morozumi and A. I. Sanda, Phys. Lett. B218 (1989) 343.

[4] B. Grinstein, M. J. Savage and M. B. Wise, Nucl. Phys. B319 (1989) 271.

[5] C. Dominguez, N. Paver and Riazuddin, Phys. Lett. B214 (1988) 459.

[6] N. G. Deshpande, J. Trampetic and K. Ponose, Phys. Rev. D39 (1989) 1461.

[7] W. Jaus and D. Wyler, Phys. Rev. D41 (1990) 3405.

[8] P. J. O'Donnell and H. K. Tung, Phys. Rev. D43 (1991) 2067.

[9] N. Paver and Riazuddin, Phys. Rev. D45 (1992) 978.

[10] A. Ali, T. Mannel and T. Morozumi, Phys. Lett. B273 (1991) 505.

[11] A. Ali, G. F. Giudice and T. Mannel, Z. Phys. C67 (1995) 417.

[12] C. Greub, A. Ioannissian and D. Wyler, Phys. Lett. B346 (1995) 145;

D. Liu Phys. Lett. B346 (1995) 355;

G. Burdman, Phys. Rev. D52 (1995) 6400;

Y. Okada, Y. Shimizu and M. Tanaka hep-ph/9704223.

[13] A. J. Buras and M. Münz, Phys. Rev. D52 (1995) 186.

[14] N. G. Deshpande, X. -G. He and J. Trampetic, Phys. Lett. B367 (1996) 362.

[15] T. M. Aliev, M. Savc1, Phys. Lett. B452 (1999) 318;

T. M. Aliev, A. Özpineci, H. Koru and M. Savc1, Phys. Lett. B410 (1997) 216.

[16] J. L. Hewett, Phys. Rev. D53 (1996) 4964.

[17] T. Inami and C. S. Lim, Prog. Theor. Phys. D65 (1981) 297; D65 (E) 1772.

[18] F. Kruger, L. M. Sehgal, Phys. Lett. B380 (1996) 199.

[19] S. Fukae, C. S. Kim and T. Yoshikawa, Phys. Rev. D61 (2000) 074015.

[20] R. Casalbuoni, A. Deandra, N. Di Bartolemo, R. Gatto and G. Nardulli, Phys. Lett. B312 (1993) 315.

[21] P. Colangelo, F. De Fazio, P. Santorelli and E. Scrimieri, Phys. Rev. D53 (1996) 3672; Erratum, ibid. D57 (1998) 3186.

[22] W. Jaus and D. Wyler, Phys. Rev. D41 (1990) 3405. 
[23] W. Roberts, Phys. Rev. D54 (1996) 863.

[24] T. M. Aliev, A. Özpineci and M. Savc1, Phys. Rev. D56 (1997) 4260.

[25] P. Ball and V. M. Braun, Phys. Rev. D58:094016, 1998.

[26] S. Fukae, C. S. Kim, T. Morozumi and T. Yoshikawa, Phys. Rev. D59 (1999) 074013.

[27] B. Grinstein, M. Savage and M. Wise, Nucl. Phys. B319 (1989) 271.

[28] S. Glashow and S. Weinberg, Phys. Rev. D15 (1977) 1958.

[29] T. P. Cheng and M. Sher, Phys. Rev. D35 (1987) 3489; ibid D44 (1991) 1461.

[30] W. S. Hon, Phys. Lett. B296 (1992) 179.

[31] A. Antaramian, L. Hall and A. Rasin, Phys. Rev. Lett. 69 (1992) 1871.

[32] L. Hall and S. Weinberg, Phys. Rev. D48 (1993) 979.

[33] M. Savage, Phys. Lett. B296 (1991) 135.

[34] D. Atwood, L. Reina and A. Soni, Phys. Rev. D55 (1997) 3156.

[35] L. Wolfenstein and Y. L. Wu, Phys. Rev. Lett. 73 (1994) 2809.

[36] D. Bowser-Chao, K. Cheung, W-Y. Keung, Phys. Rev. D59 (1999) 115006.

[37] T. M. Aliev, E. O. İltan, Phys. Rev. D58 (1998) 095014.

[38] T. M. Aliev, E. O. İtan, J. Phys. G25 (1999) 989.

[39] E. O. İltan, Phys. Rev. D60 (1999) 034023.

[40] T. M. Aliev, M. Savc1, Phys. Rev. D60 (1999) 014005.

[41] Yuan-Ben Dai, Chao-Shang Huang and Han-Wen Huang, Phys. Lett. B390 (1997) 257.

[42] A. J. Buras and M. Münz, Phys. Rev. D52 (1995) 4223.

[43] M. Misiak, Nucl. Phys. B393 (1993) 23; Erratum, ibid. B439 (1995) 461.

[44] Z. Ligeti, M. B. Wise, Phys. Rev. D53 (1996) 4937. 


\section{Figure captions}

Fig. 1 The dependence of the CP-violating asymmetry $A_{C P}$ of the $\tau$ lepton on $q^{2}$ and on the weak phase $\phi$ in model III.

Fig. 2 The dependence of the averaged CP asymmetry $\left\langle A_{C P}\right\rangle$ of the $\tau$ lepton on the weak phase $\phi$ in model III, taking into account short and long distance contributions.

Fig. 3 The dependence of the longitudinal polarization $P_{L}$ of the $\tau$ lepton on $q^{2}$ and on the weak phase $\phi$ in model III, taking into account only the short distance contribution in $C_{9}^{e f f}$.

Fig. 4 The dependence of the averaged longitudinal polarization $\left\langle P_{L}\right\rangle$ of $\tau$ lepton on the weak phase $\phi$, taking into account short and long distance contributions.

Fig. 5 The dependence of the transversal polarization $P_{T}$ of the $\tau$ lepton on $q^{2}$ and on the weak phase $\phi$ in model III, taking into account only the short distance contribution in $C_{9}^{e f f}$.

Fig. 6 The dependence of the normal polarization $P_{N}$ of the $\tau$ lepton on $q^{2}$ and on the weak phase $\phi$ in model III, taking into account only the short distance contribution in $C_{9}^{e f f}$.

Fig. 7 The dependence of the averaged transversal polarization $\left\langle P_{T}\right\rangle$ of the $\tau$ lepton on the weak phase $\phi$, taking into account short and long distance contributions.

Fig. 8 The dependence of the averaged normal polarization $\left\langle P_{N}\right\rangle$ of the $\tau$ lepton on the weak phase $\phi$, taking into account short and long distance contributions. 


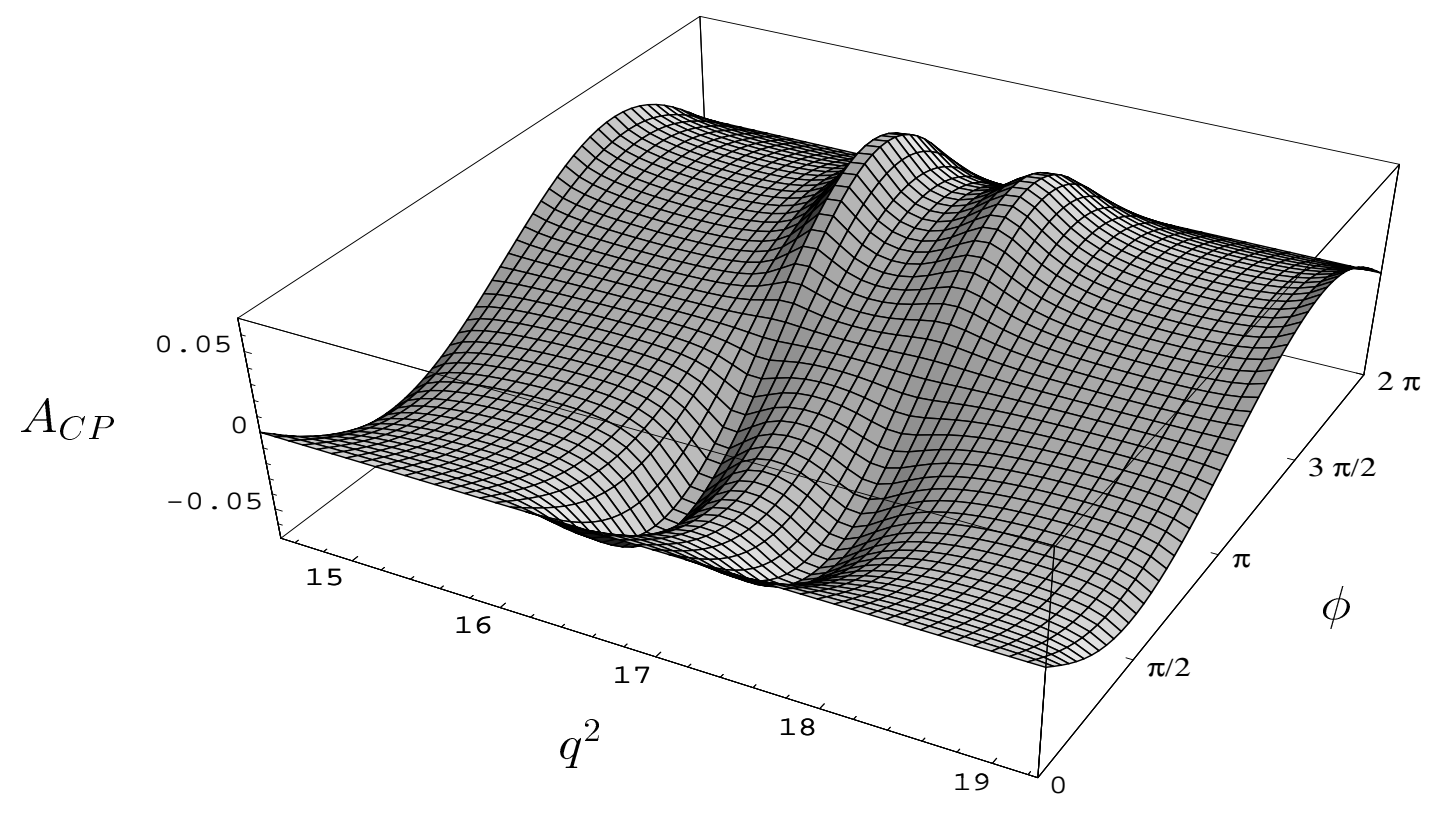

Figure 1:

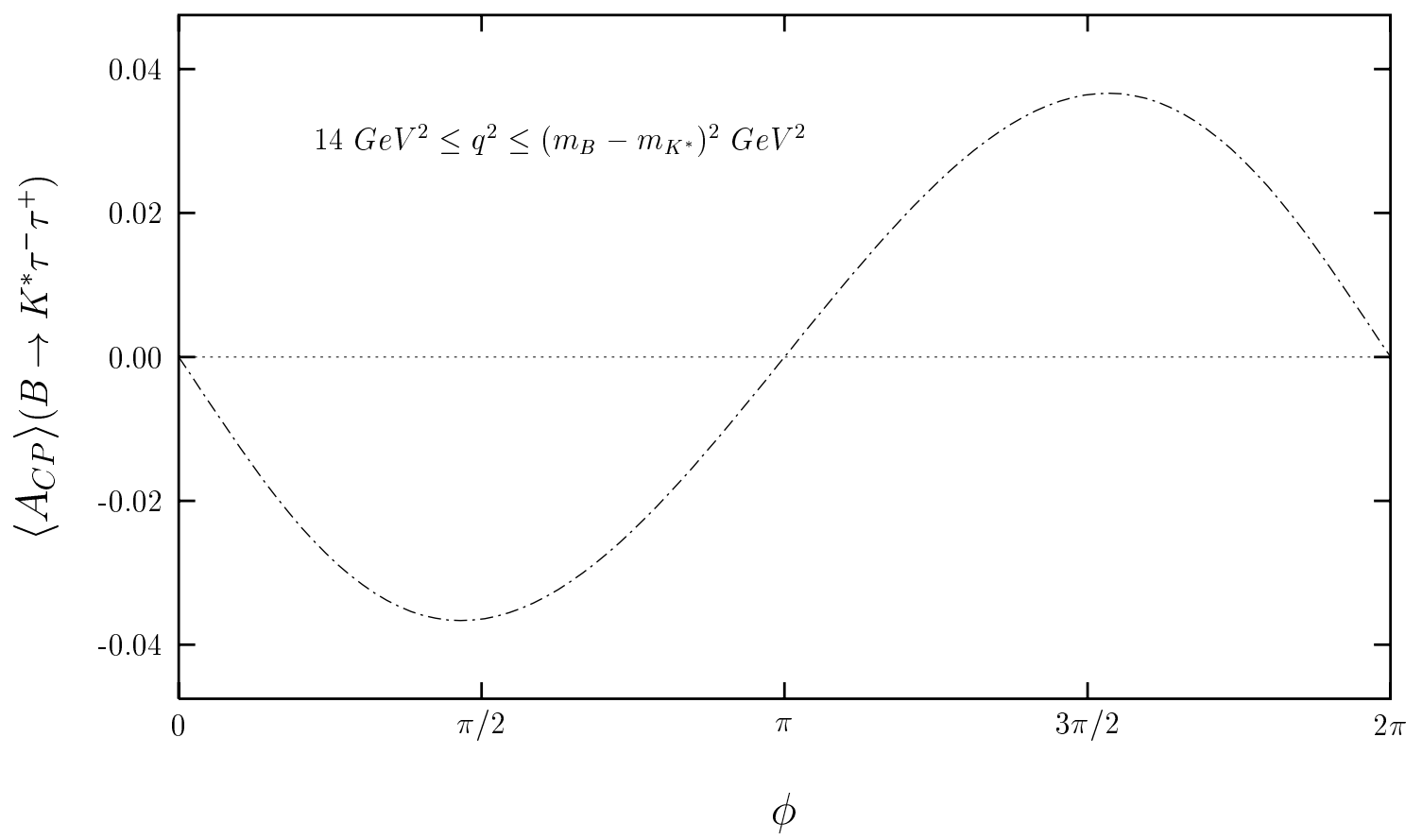

Figure 2: 


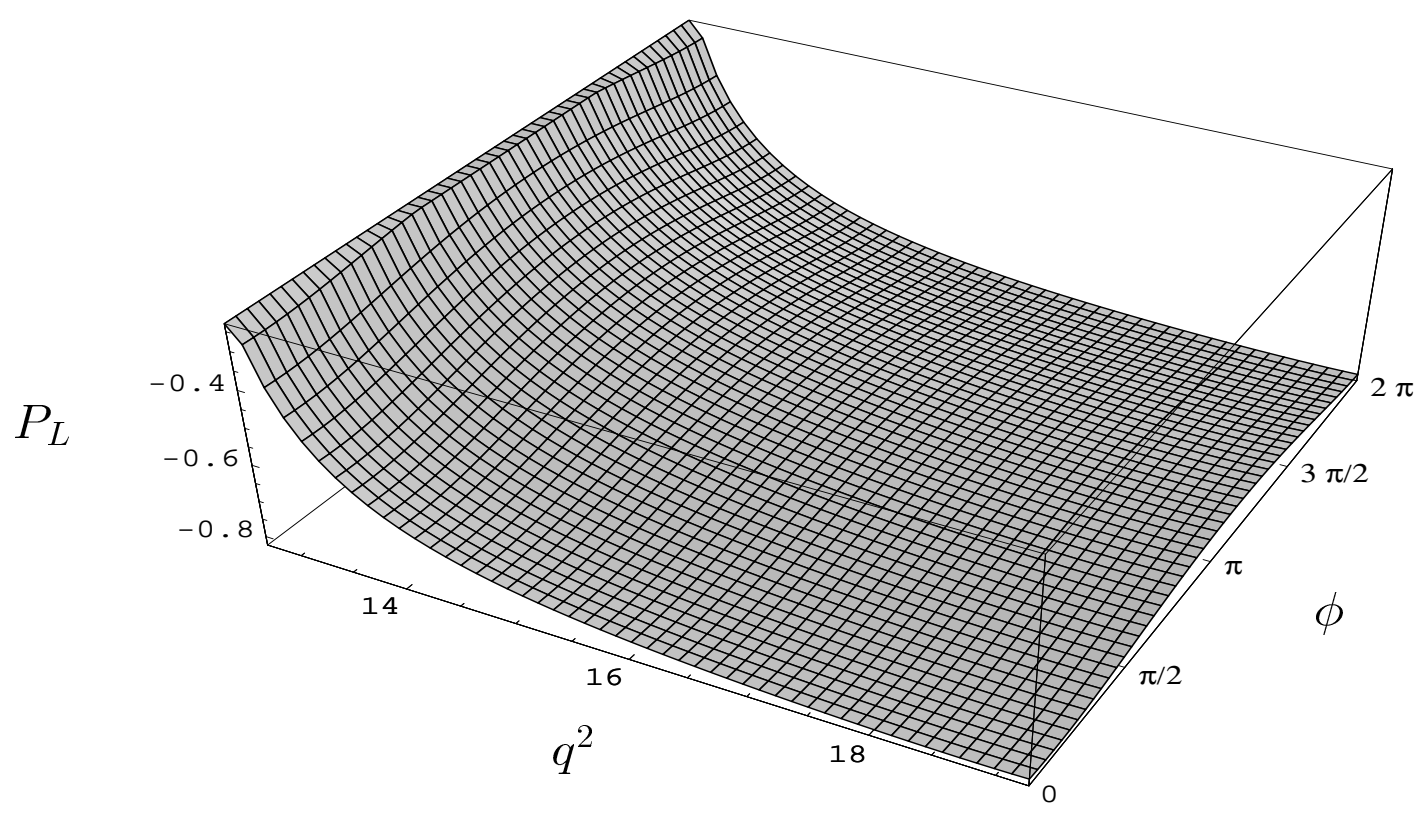

Figure 3:

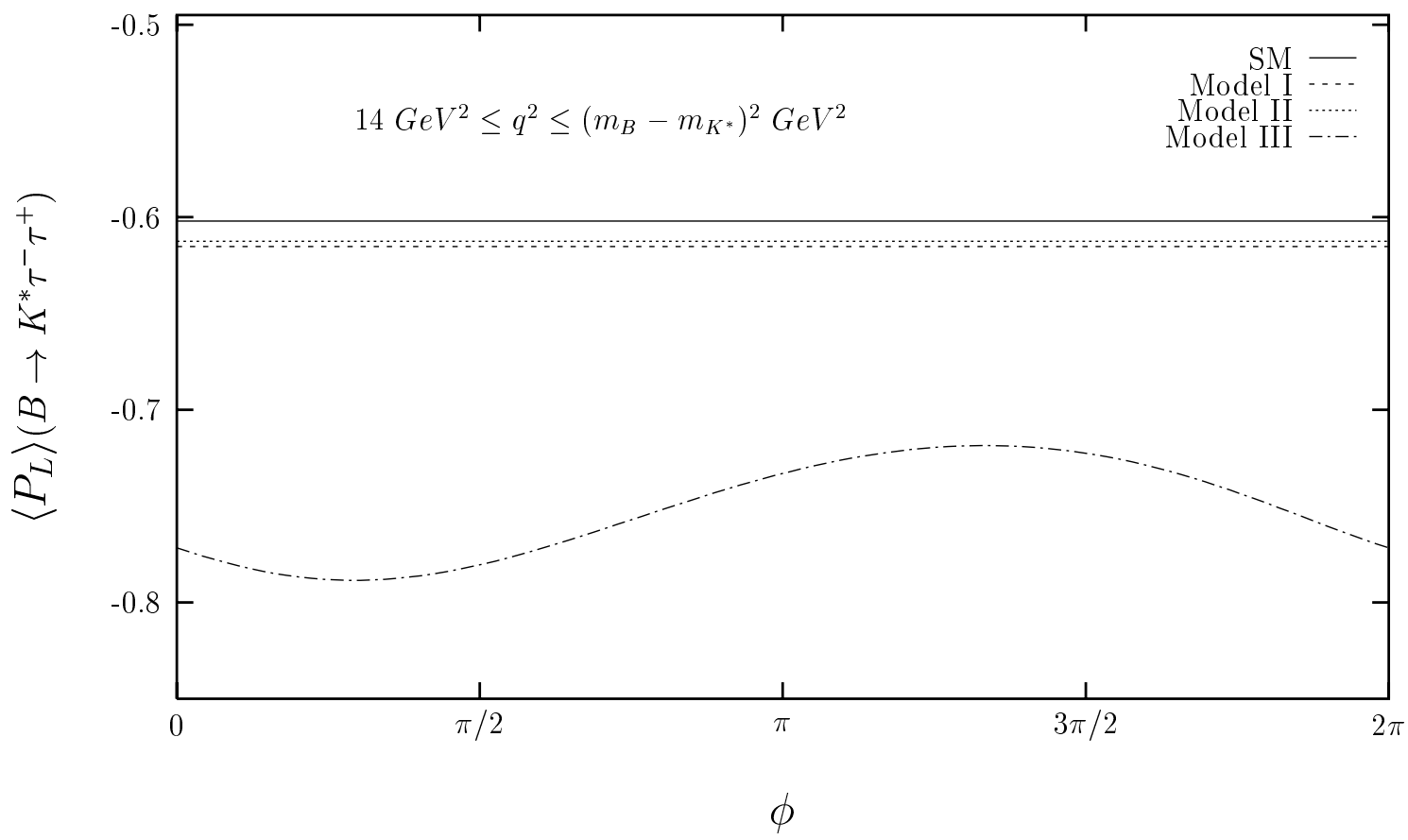

Figure 4: 


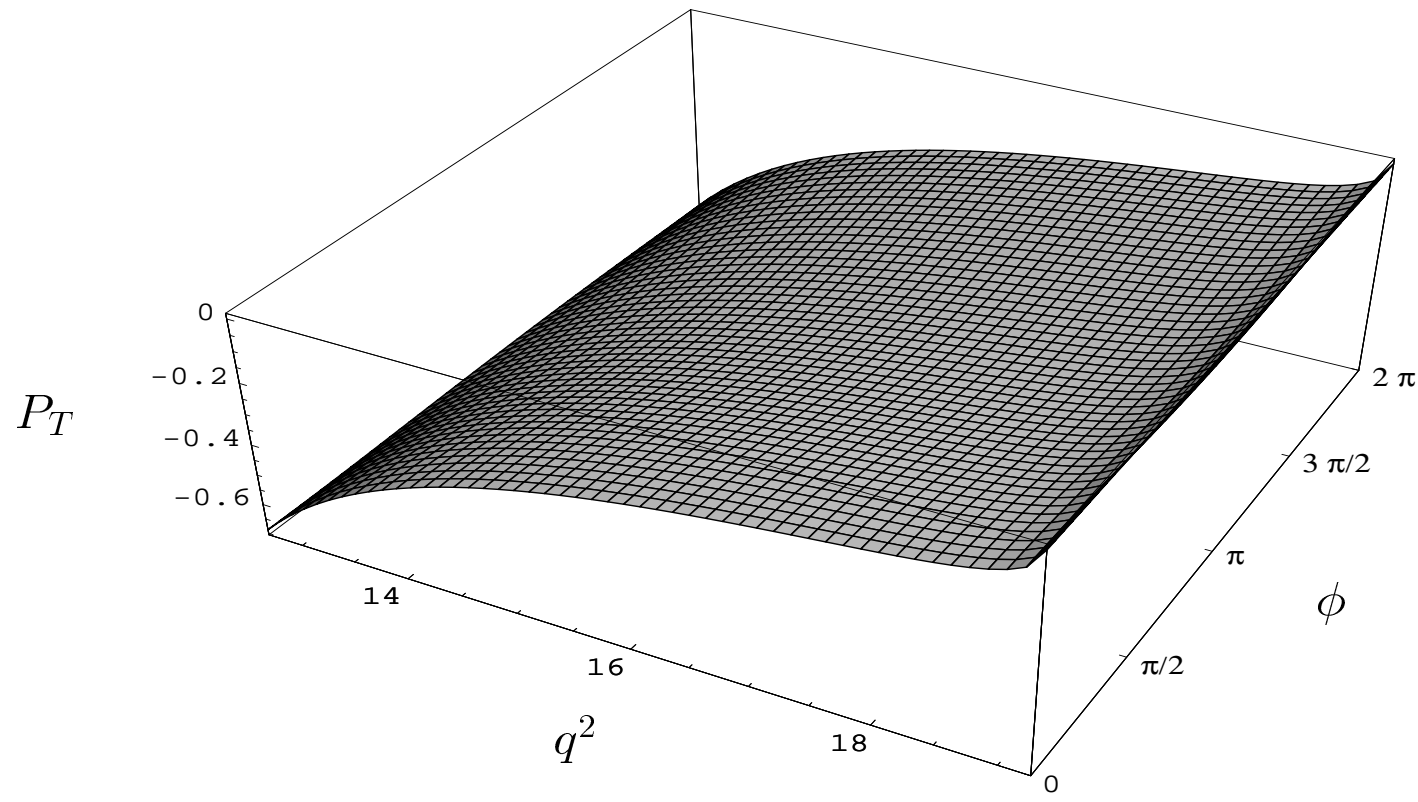

Figure 5:

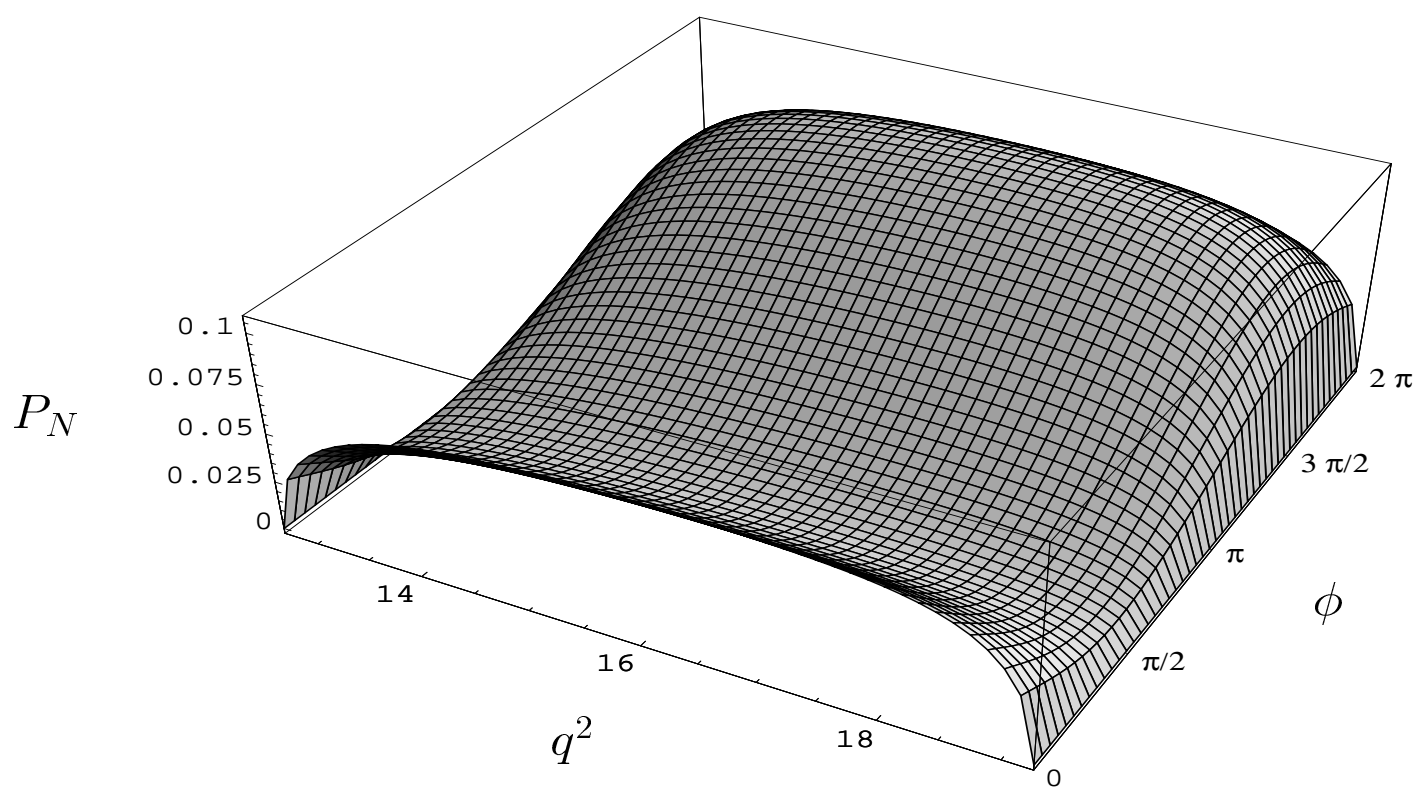

Figure 6: 


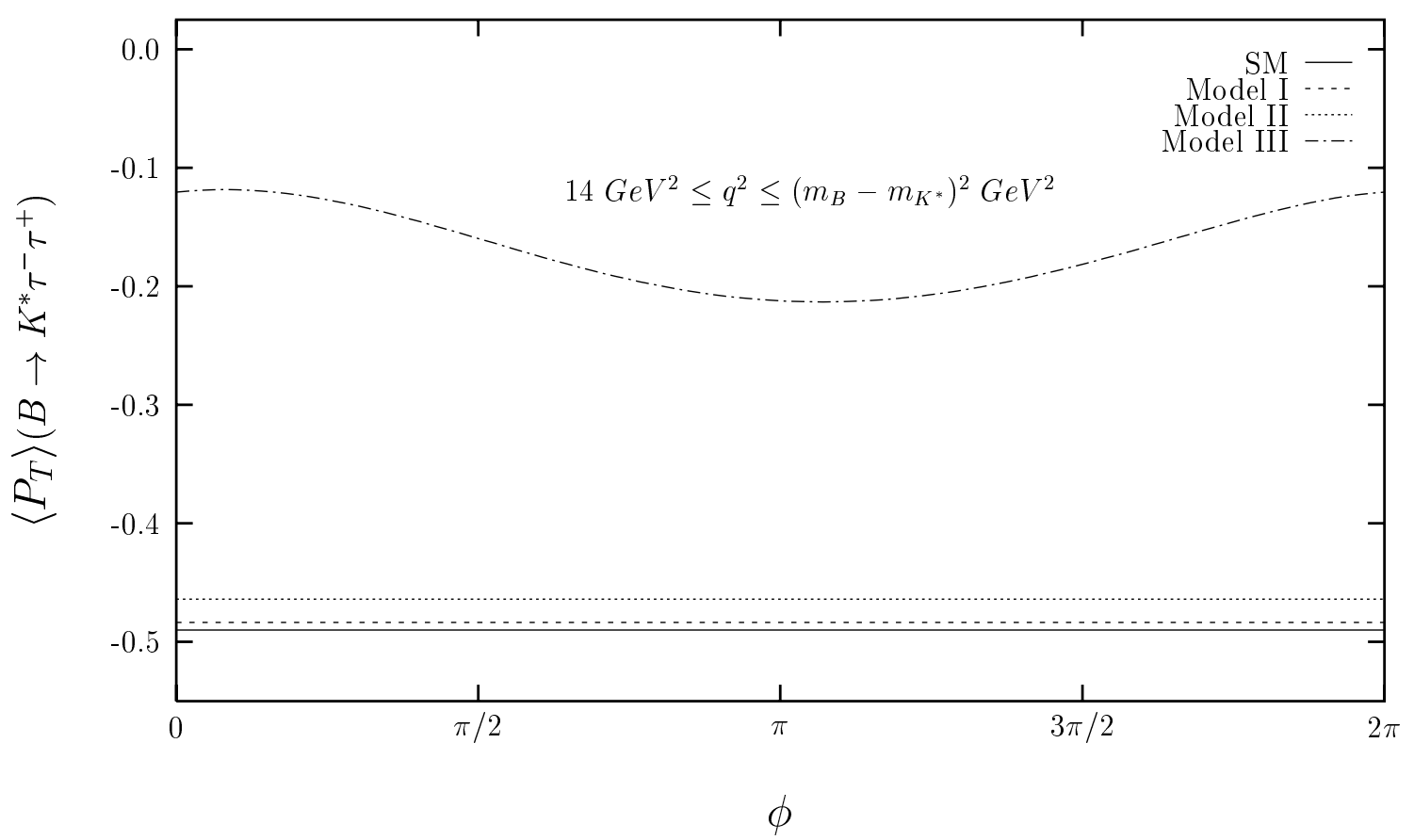

Figure 7:

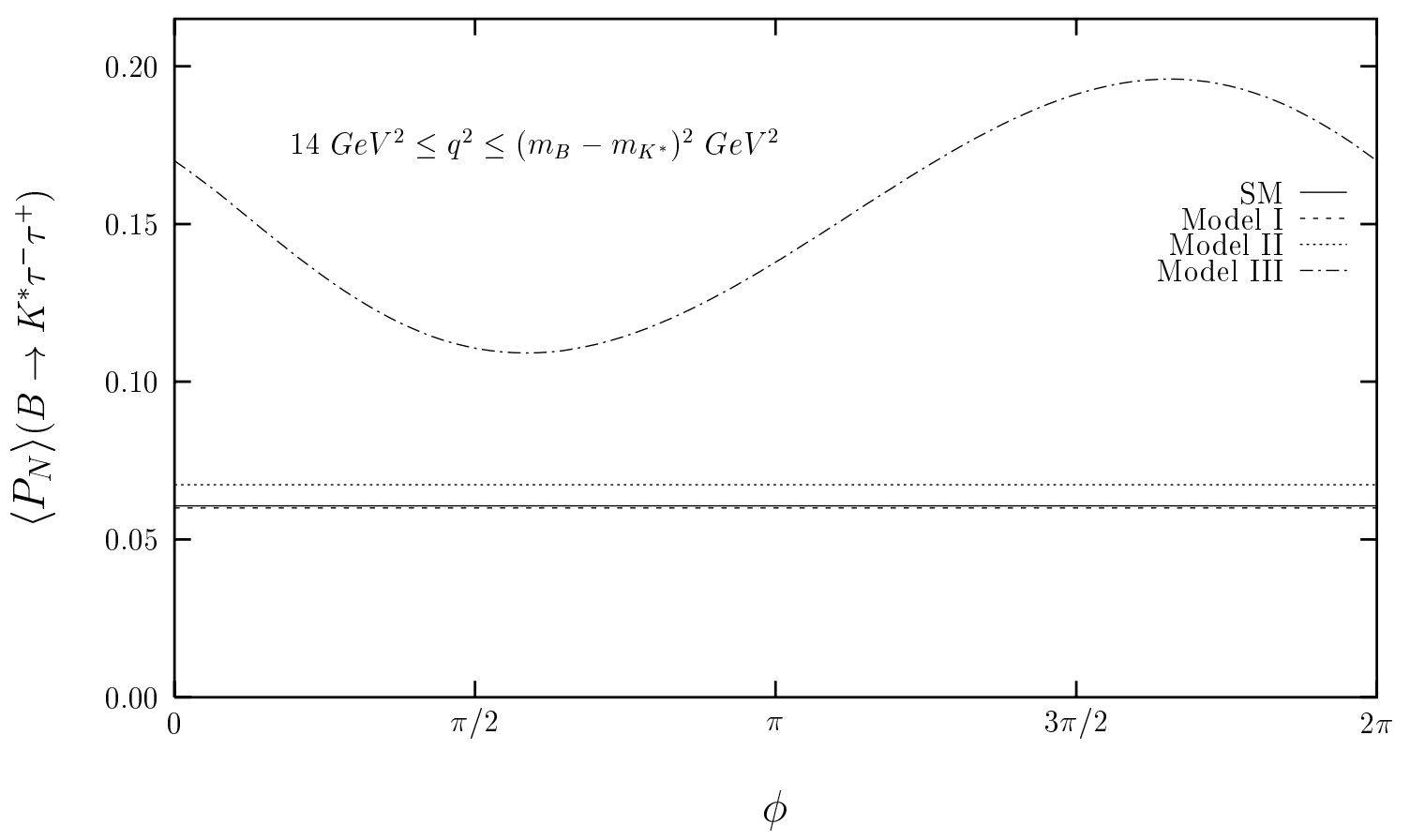

Figure 8: 